\title{
Protocolo para captura de señales electromiográficas producidas por músculo uterino en gestantes para predicción de parto pretérmino
}

Protocol for Capturing Electromyographic Signals Produced by Uterine Muscle in Pregnant Women for Predicting Preterm Delivery

Marco Antonio Chamorro Lucero ${ }^{1}$ María Fernanda Acosta Romo ${ }^{2}$

OPEN ACCESS

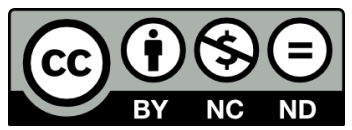

Copyright:

(02021. La revista Ingenierías USBmed proporciona acceso abierto a todos sus contenidos bajo los términos de la licencia creative commons Atribución no comercial SinDerivar 4.0 Internacional (CC BY-NC-ND 4.0)

Tipo de artículo: Investigación.

Recibido: 05-13-2020.

Revisado: 31-07-2020.

Aprobado: 22-10-2020.

Doi: $10.21500 / 20275846.4715$

Referenciar así:

M. A. Chamorro-Lucero y M. F Acosta-Romo, "Protocolo para captura de señales electromiográficas producidas por músculo uterino en gestantes para predicción de parto pretérmino," Ingenierías USBMed, vol. 12, no. 1, pp. 19-24, 2021.

\section{Disponibilidad de datos:}

todos los datos relevantes están dentro del artículo, así como los archivos de soporte de información.

\section{Conflicto de intereses:}

los autores han declarado que no hay conflicto de intereses.

Editor: Andrés Felipe Hernández. Universidad de San Buenaventura, Medellín, Colombia.

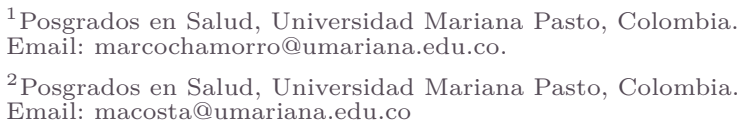

Resumen. El objetivo de la presente investigación fue diseñar un protocolo de proceso de captura de señales electrohisterográficas (EHG), para predicción de parto pretérmino. El estudio fue de tipo exploratoriodescriptivo, propositivo. Para el diseño se realizó revisión documental de artículos científicos investigativos de corte cualitativo y cuantitativo, en inglés y español, consultados en fuentes de información biomédica especializados como el MEsH y DeCS y en bases de datos como: PubMed, Scielo, IEEE, entre otras. Las expresiones de búsqueda fueron: electromiografía, músculo uterino y protocolo. No se encontraron protocolos específicos para electrohisterografía, por lo que se tomaron publicaciones referentes a protocolos de electromiografía en general, encontrándose un total de 193, de las que se seleccionaron 23, publicadas entre 2012 y 2020. Para el análisis, se utilizó el método de investigación denominado revisión integradora. Resultados: el protocolo plantea criterios que deben tenerse en cuenta antes y durante el procedimiento, equipos y materiales requeridos y contraindicaciones para la captura de registro de señales electrohisterográficas. Conclusión: El protocolo de captura de señales EHG permite estandarizar el procedimiento para obtener señales óptimas para la predicción de parto pretérmino.

Palabras Clave. Electromiografía, Miometrio, protocolo clínico (DeCSBireme).

Abstract. The objective of this research was to design a protocol for the capture of electrohysterographic signals, for the prediction of preterm delivery.The study was exploratory - descriptive, purposeful. For the design, a documentary review of qualitative and quantitative scientific research articles was carried out, in English, Spanish, they were consulted in sources of biomedical information with words from specialized thesauri such as MEsH and DeCS, they were consulted in databases: PubMed, Scielo, IEEE, among others. The search expressions were: Electromyography, uterine muscle and protocol. No specific protocols for uterine electrohysterography were found, which is why the publications referring to electromyography protocols in general were taken, finding a total of 193 publications, of which 23 articles were selected, published between the years 2012 to 2020. For the analysis the research method called integrative review was used. Results: The protocol establishes the criteria that must be taken into account before and during the procedure, the equipment and materials required, and contraindications, to capture the record of electrohysterographic signals. Conclusion: The EHG signal capture protocol allows standardizing the procedure to obtain optimal signals for predicting preterm delivery.

Keywords. Electromyography, Myometrium, Clinical Protocols. 


\section{Introducción}

El parto pretérmino se produce por la aparición de contracciones uterinas, después de la semana 22 y antes de la semana 37 [1]. Cada año, nacen en el mundo 15 millones de niños prematuros, con una incidencia mundial de aproximadamente $9.6 \%$; la tasa de nacimientos prematuros oscila entre el $5 \%$ y el $18 \%$ [2]. En los últimos años, se han incrementado los nacimientos de partos prematuros, a pesar de los avances en intervenciones médicas, siendo el responsable del $75 \%$ de la mortalidad neonatal [3]. Por esta razón, se pretenden encontrar nuevas técnicas no invasivas como alternativas que permitan diagnosticar con mayor precisión el parto pretérmino, con el fin de disminuir la morbimortalidad neonatal y los costos asociados a la atención de un recién nacido prematuro, ya que actualmente se disponen algunos métodos que no han sido determinantes en este diagnóstico.

En la actualidad, el avance tecnológico ha permitido adelantos importantes en el campo de la salud en favor de atender las necesidades del ser humano. Por medio de sensores y análisis de datos, se desarrollan herramientas que mejoran los servicios de salud en el monitoreo de enfermedades y caracterización de patrones para apoyar los diagnósticos médicos. El análisis del registro de superficie de la actividad mioeléctrica uterina (electrohisterografía) y su electrohisterograma, son algunas de las nuevas herramientas de ayuda diagnóstica, que evalúan las contracciones y el estado electrofisiológico del útero, se definen como una serie de registros de la actividad mioeléctrica uterina; esta técnica no es invasiva y es capaz de distinguir contracciones efectivas y no efectivas al parto [4], se realiza a través de electrodos de superficie, los cuales se ubican en el abdomen de la gestante.

La elaboración de este protocolo surge del proyecto de investigación titulado: "Desarrollo de un sistema de captura de señales Electrohisterográficas (EHG) en gestantes con trabajo de Parto Pretérmino, Hospitalizadas en el servicio de Ginecobstetricia", además se justifica desde la necesidad de estandarizar el proceso de recolección de señales electrohisterográficas en gestantes.

El protocolo plantea los criterios que deben tenerse en cuenta antes y durante el procedimiento, los equipos y materiales requeridos, ubicación de sensores o electrodos de superficie sobre la piel de la paciente y contraindicaciones, para toma de registro de las señales EHG; lo mencionado asume un abordaje desde la evidencia científica, mediante la metodología de revisión integradora.

\section{Materiales y métodos}

El estudio fue de tipo exploratorio-descriptivo, propositivo, se realizó revisión documental de corte cualitativo y cuantitativo: las unidades de análisis fueron ar- tículos científicos escritos en inglés y español, consultados en fuentes electrónicas de información biomédica y con palabras de tesauros especializados como el MEsH y el DeCS; se consultaron bases de datos como: PubMed, Scielo, IEEE, Scientific Electronic Library Online, Cochrane, Medline, Pan American Health Organization Database Search, (OPS), Medline, Lilacs, Scielo Colombia, Dialnet, Pro-quest, y Google Académico. Las expresiones de búsqueda fueron: electromiografía, músculo uterino y protocolo, que se combinaron para realizar la búsqueda. Inicialmente se filtró la información por año desde el 2015 a 2020; sin embargo, debido a la escasa información, se amplió el periodo de búsqueda entre los años 2012 a 2020. En la revisión bibliográfica no se encontraron protocolos específicos para electrohisterografía, razón por la que se tomaron las publicaciones referentes a protocolos de electromiografía en general, que fueron utilizados para el desarrollo de la propuesta de protocolo. Para el análisis se utilizó el método de investigación denominado revisión integradora, que permitió procesar la información de los documentos revisados de manera precisa y ordenada en el proceso de búsqueda, evaluación crítica y síntesis de las evidencias sobre el tema investigado

El presente protocolo fue evaluado por dos expertos: uno del área de Ingeniería Biomédica y otro del área de la Salud, especialista en Ginecología; además, se tuvieron en cuenta los principios éticos de la Resolución 008430 de octubre 4 de 1993. Asimismo, se respetaron los principios éticos fundamentales que regulan la práctica clínica como son: no maleficencia, justicia, autonomía y beneficencia. De igual forma, se respetó la autonomía de las personas (consentimiento informado) y la confidencialidad, asegurando la protección de la identidad de los participantes. El estudio contó con la aprobación del Comité de Ética y Bioética de la Universidad Mariana, por medio del acuerdo 093 del 17 de Mayo de 2017.

\section{Resultados}

De la revisión bibliográfica, se tomaron publicaciones referentes a protocolos de electromiografía en general, se analizaron y se seleccionaron los documentos referentes a protocolos de captura de señales electromiográficas. Finalmente se tomaron 23 artículos, como se muestra en la Figura 1.

\section{A. Descripción del Protocolo}

Generalmente, la electromiografía de superficie no tiene contraindicaciones, pero es importante tener en cuenta algunos factores que pueden alterar los resultados del estudio. Para la toma de las señales se determinaron algunos criterios en los que no es posible realizar este procedimiento, tales como: gestantes que se encuentren en estado de agitación (estrés, tensión), gestantes con diagnóstico de obesidad y gestantes con marcapasos; 


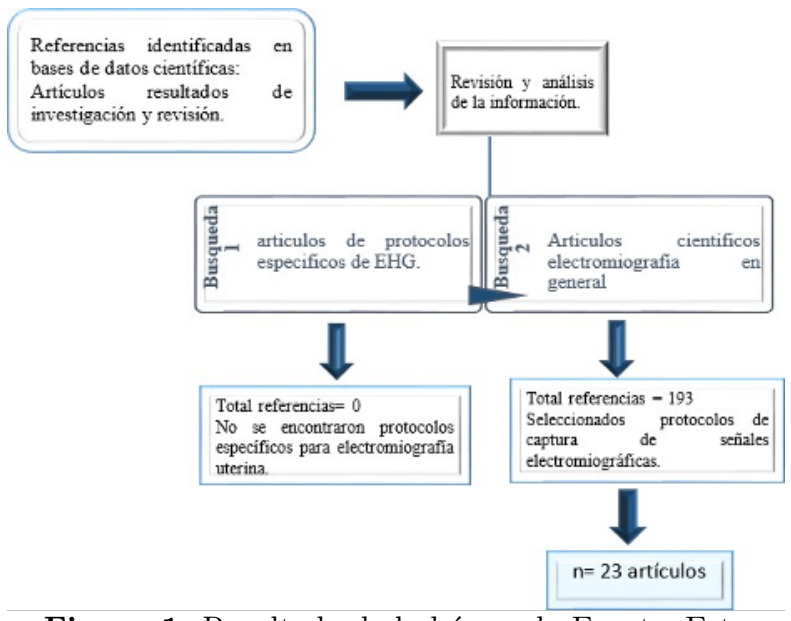

Figura 1. Resultado de la búsqueda Fuente: Esta investigación

además, puede estar contraindicado para pacientes con infecciones cutáneas de gran extensión localizadas en abdomen, debido al riesgo de propagar la infección a otras zonas de la piel, dado el uso de los electrodos. Los medicamentos como los relajantes musculoesqueléticos, los colinérgicos y los anticolinérgicos también pueden influir en los resultados de la electrohisterografía.

Resulta importante, por otro lado,disponer de buenas condiciones ambientales, con el fin de adquirir bioseñales sin ruido electromagnético. Se recomienda que en el momento de tomar la captura de las señales no se deben tener cerca radioteléfonos, transmisores de radiofrecuencia, celulares u otros dispositivos. Además, se debe brindar a la paciente un espacio adecuado y cómodo para la captura de dichas señales.

Para lograr la captura de la actividad eléctrica uterina, se diseñó un sistema mediante filtros y amplificadores, de acuerdo con las características de la actividad eléctrica del musculo uterino, utilizando diferentes tarjetas de adquisición de bioseñales disponibles en el mercado, logrando tener un mejor resultado con la ESP32, que es un SoC (System on Chip) que integra el procesador de 32 bits LX6 de doble núcleo, previamente programada desde el entorno de Arduino. Para el sistema de comunicación con el ordenador, se utilizó el software de Labview, logrando programar el tiempo de captura y la visualización en pantalla de los registros. La tarjeta desarrollada, finalmente, se conecta a la gestante mediante un sistema de 4 electrodos de superficie.

\section{B. Descripción del Procedimiento}

- Informar a la paciente en qué consiste el procedimiento que se va a realizar, incluyendo los riesgos que puedan presentarse en el desarrollo de este. Es importante mencionar que no existen limitaciones después de realizar la prueba y que, luego de finalizada, puede continuar con sus actividades normales.

- Entregar el consentimiento informado para que la pa- ciente lo lea y atender cualquier duda que tenga sobre el procedimiento.

- Brindar información sobre elementos que puedan interferir en la captura de señales como marcapasos u otros dispositivos cardiacos o electrónicos.

- Explicarle que no necesita compañía para la captura de las señales, ni estar en ayunas; posteriormente, se le solicita que descubra el abdomen. Se debe evitar el uso de cremas y productos de higiene personal en el abdomen.

- Disponer de electrodos desechables no invasivos o de superficie, de cloruro de plata, bipolares, que pueden presentar diámetros desde 20 hasta $50 \mathrm{~mm}$. Se recomienda usar de alrededor de $24 \mathrm{~mm}$.

- Disponer de la tarjeta de adquisición para el registro de las diferentes señales electrohisterográficas.

- Preparar la piel del abdomen de la gestante, higienizando con una gasa y alcohol los puntos escogidos previamente, donde se captarán las señales alrededor del ombligo.

- Aplicar gel conductivo a los electrodos para el registro.

- Conectar los electrodos a los broches de las puntas o conectores cocodrilo de tamaño M, con terminal hembra a presión, estos se conectan hacia amplificadores operacionales en la tarjeta y éstos a su vez a la PC a través de un puerto USB. La visualización de la señal se realiza a través de LabView.

- Configurar el software para grabar la captura.

- Conectar la fuente de alimentación de la tarjeta y el computador.

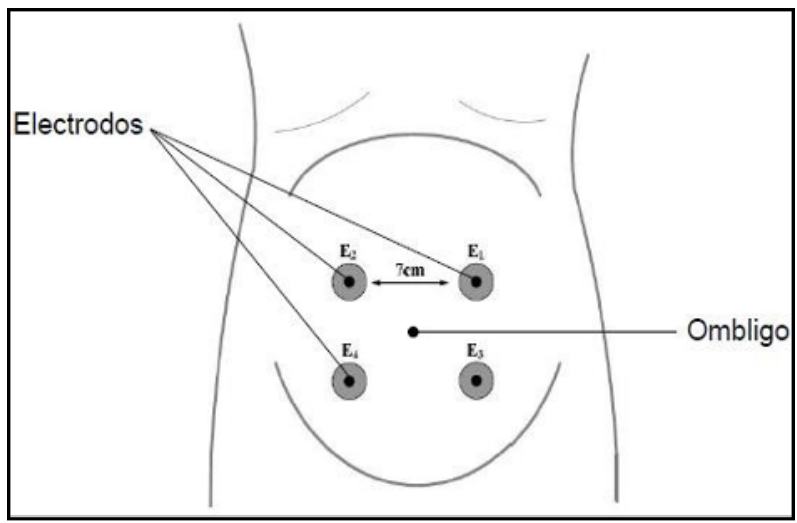

Figura 2. Disposición de electrodos superficiales en el abdomen. Ubicación centrada en el ombligo del paciente [5]

- Ubicar los electrodos superficiales en los puntos especificados, tomar el punto medio del ombligo para formar un cuadro en el que se ubicarán los electrodos para registrar las señales de EEG. El primer electrodo (E1) se ubica a $3.5 \mathrm{~cm}$ por encima del ombligo y a 3.5 $\mathrm{cm}$ hacia la derecha del ombligo, el segundo electrodo (E2) está ubicado a $3.5 \mathrm{~cm}$ por encima y a $3.5 \mathrm{~cm}$ a la izquierda del ombligo; el tercer electrodo se encuentra ubicado a $3.5 \mathrm{~cm}$ por debajo del ombligo y $3.5 \mathrm{~cm}$ 
hacia la derecha, y finalmente, el cuarto electrodo se encuentra ubicado a $3.5 \mathrm{~cm}$ por debajo del ombligo y $3.5 \mathrm{~cm}$ hacia la izquierda (ver Figura 2).

- La captura se empezará con la gestante en estado basal (acostada) y relajada, debido al ruido generado por el movimiento de los electrodos y la piel del paciente, evitando la interferencia de la señal [6]. Deberá estar en una posición cómoda a una distancia de $30 \mathrm{~cm}$ de la pantalla del computador para empezar el registro, que durará aproximadamente 30 minutos.

- Iniciar la captura de las señales, registrando los eventos relevantes durante la grabación con el fin de documentar cualquier anomalía en el proceso. Cuando se finalice la captura, se detiene la grabación de las señales. - Retirar los electrodos de la paciente y proceder a la limpieza de la piel abdominal.

- Dejar cómoda a la paciente.

\section{Discusión}

Para la captura de la Electromiografía de superficie (SEMG), existen diferentes formas y procedimientos, como la presentada por la International Society of Electrophysiology and Kinesiology (ISEK), que ha establecido protocolos de registro para que se puedan capturar, interpretar y comparar los resultados de la SEMG [7]. Estos protocolos, así como los presentados en el proyecto SENIAM (Surface EMG for Non-Invasive Assessment of Muscles), relacionan la SEMG de acuerdo al músculo sobre el que se está trabajando. Sin embargo, en la revisión bibliográfica realizada no se encontró ninguno para SEMG del músculo uterino, razón por la que se tomaron como base algunos protocolos establecidos para músculos lisos, que son similares al músculo uterino.

En el presente estudio se establece que, para la adquisición de señales electrohisterográficas, se deben utilizar electrodos no invasivos o de superficie, que se adhieren directamente en la piel del abdomen de la gestante [8]; el tipo de electrodos utilizados en el registro de estas señales son los de cloruro de plata $(\mathrm{Ag} / \mathrm{AgCl})$, compuestos por un núcleo de plata $(\mathrm{Ag})$ y rodeado por un revestimiento de cloruro de plata $(\mathrm{AgCl})$ [9]. Resultados de estudios indican que los electrodos bipolares y tripolares tienen una alta sensibilidad local en comparación con otro tipo de electrodos [10], garantizando la confiabilidad y calidad en el diagnóstico [6]. Además, estos son ideales debido a la estabilidad que presenta al contacto con el gel empleado en este tipo de pruebas [9], [11]. Antes de adherir los electrodos se recomienda preparar la piel, realizando un proceso de limpieza y aplicando gel conductivo a los electrodos [12].

De igual manera, se establece que este procedimiento no se recomienda que sea realizado en gestantes con obesidad, debido a que la cantidad excesiva de grasa entre el músculo y la piel reduce la amplitud de las señales; también, se debe tener precaución en retirar de la paciente fuentes de radiación electromagnética, ya que pueden causar interferencia durante la grabación de las señales [13], [14].

En relación a la disposición de los electrodos superficiales sobre el abdomen, la mayoría de los estudios electrohisterográficos se han focalizado en el registro bipolar, obtenido mediante la colocación de dos electrodos monopolares sobre la pared abdominal. La ventaja [15] del registro bipolar [16] estriba en la eliminación de las interferencias, que suelen quedar registradas en ambos registros monopolares [16]. Algunos autores indican la colocación de los electrodos en forma de una línea vertical sobre el eje medio del abdomen, con la intención de detectar la propagación de las contracciones [17]. Estudios han demostrado que los electrodos debajo del ombligo parecen tener mayor potencial para diferenciar el parto prematuro del parto a término: estos hallazgos fueron confirmados por Ren, Yao, Li, Valdes-Sosa y Kendrick [18], quienes mostraron que el canal 3 es mejor que los canales 1 y 2 . Por lo tanto, las grabaciones con electrodos debajo del ombligo suministran mayor predicción de riesgo de parto prematuro.

De acuerdo con diversas investigaciones, se puede mencionar que aún no existe una forma estandarizada de ubicación de los electrodos [19], por consiguiente, para el estudio actual, se diseñó el protocolo optando por sugerir que el procedimiento de captura de señales EHG se realice colocando los electrodos en la región abdominal baja, ya que esta ubicación es considerada la más precisa para una óptima adquisición de estas señales en gestantes [20]. Se prepara la piel de forma cuidadosa, garantizando la calidad de las señales y facilitando, posteriormente, su interpretación [6]. En el siguiente paso, se aplica gel conductor sobre la superficie del electrodo con el fin de reducir la impedancia de la interfaz electrodo-piel [21].

Los electrodos proporcionan el medio para conducir una corriente a través de la interfaz entre el cuerpo y la tarjeta desarrollada, sirviendo como transductores, y los filtros eliminan los ruidos y otras señales que puedan interferir en la captura de las señales EHG, que ocupa un espectro de frecuencia en el ancho de banda de 0.1 a $3 \mathrm{~Hz}$; además, parte de la energía de la señal EHG está en un ancho de banda inferior a $0.1 \mathrm{~Hz}$, sin embargo, el ancho de banda por debajo de $0.033 \mathrm{~Hz}$ es probablemente causado por artefactos mecánicos y no por actividad eléctrica en el útero [17].

Para el registro de señales EHG, se implementó un hardware de acondicionamiento de las señales captadas por los electrodos bipolares para dos canales [20], conectados a un software (Labview) para la visualización de las señales en el computador mediante una aplicación que permite determinar el tiempo de grabación, cambiar la frecuencia y visualizar en forma instantánea los datos capturados. En este apartado se hace 
una recomendación sobre la precaución al conectar el ordenador al tomacorriente: verificar que esté en buen estado para evitar cualquier riesgo eléctrico. Posteriormente, revisar el estado de las baterías que se utilizan para la etapa de amplificación, que deben estar con la suficiente carga [6].

Se recomienda que, para iniciar el registro de la señal electrohisterográfica, la paciente se encuentre en estado de reposo, debido al ruido artefacto que se produce por los movimientos de los electrodos y la piel del paciente, ya que esto puede generar interferencia en las señales capturadas [6]. Finalmente, se determinó que el tiempo de grabación debe ser de 30 a 40 minutos, de acuerdo con las revisiones realizadas [17], y también tomando como referencia los métodos de monitorización fetal que se realizan actualmente [22].

\section{Conclusiones}

El presente protocolo clínico de captura de señales EHG para predicción de parto prematuro, permite estandarizar el proceso que se debe seguir antes y durante el procedimiento, al igual que equipos y materiales requeridos y contraindicaciones que deben tener en cuenta para la toma de señales EHG en gestantes. Su diseño se realizó desde una mirada de la Ingeniería clínica para apoyar el trabajo de los profesionales del área de la salud en un lenguaje claro y sencillo, teniendo en cuenta la evidencia científica; su aplicación es útil para minimizar las posibles variaciones que puedan interferir en el proceso de toma de señales electrohisterográficas, lo que incide en el mejoramiento de la calidad de atención a mujeres gestantes.

En la revisión bibliográfica, a pesar de que se encontraron artículos de protocolos de electromiografía, no se encuentra suficiente evidencia de protocolos específicos para captura de señales EHG, por lo que se propone el presente protocolo con el fin de estandarizar criterios en el procedimiento de captura de señales EHG para la predicción de parto pretérmino.

\section{Referencias}

[1] E. Huertas Tacchino, "Parto pretérmino: causas y medidas de prevención," Revista peruana de Ginecología y obstetricia, vol. 64, n. $^{\circ} 3$, págs. 399-404, 2018.

[2] Organización Mundial de la Salud, "Nacimientos prematuros," 19 febrero 2018. [Online].

Available: https://www.who.int/es/news-room/ fact-sheets/detail/preterm-birth.

[3] Organización Mundial de la Salud, "Mejorar la supervivencia y el bienestar de los recién nacidos," 19 septiembre 2020. [Online].

Available: https://www.who.int/es/news-room/fact-sheets/detail/newborns-reducing-mortality.
[4] J. Escalante-Gaytán, C. G. Esquivel-Arizmendi, C. I. Ledesma-Ramírez, A. C. Pliego-Carrillo, M. T. García-González y J. J. Reyes-Lagos, "Utilidad de la electrohisterografía como técnica de monitorización uterina en el ámbito clínico: revisión bibliográfica," Ginecología y obstetricia de México, vol. 87, n. ${ }^{\circ} 1$, págs. 46-59, ene. de 2019.

[5] S. M.-S. Baghamoradi, M. Naji y H. Aryadoost, Evaluation of cepstral analysis of EHG signals to prediction of preterm labor, IEEE, 2011, pp. 81-83.

[6] C. A. Alva Coras, "Procesamiento de señales de electromiografía superficial para la detección de movimiento de dos dedos de la mano," Tesis de pregrado, Lima, Universidad Ricardo Palma, 2012.

[7] R. Merletti, "Standards for Reporting EMG Data," Journal of Electromyography and kinesiology, vol. 9, n. ${ }^{\circ}$ 1, págs. III-IV, feb. de 1999.

[8] J. M. Esnaola Capa, "Caracterización de la señal electromiográfica en superficie de la musculatura de suelo pélvico en pacientes con dolor pélvico crónico tratados con toxina botulínica," Tesis de pregrado, Valencia, Universitat Politècnica de València, 2019.

[9] P. Gao, D. Hao, Y. An, Y. Wang, Q. Qiu, L. Yang, Y. Yang, S. Zhang, X. Li y D. Zheng, "Comparison of electrohysterogram signal measured by surface electrodes with different designs: A computational study with dipole band and abdomen models," Sci Rep, vol. 7, pág. 17282 , dic. de 2017.

[10] J. D. Mantilla Brito, "Diseño de un sistema de control de una mano robótica mediante señales electromiográficas," Tesis de pregrado, Universidad de Las Américas, 2019.

[11] A. C. Cayachoa Vargas y V. J. Neira Rivera, "MedElektra : módulo de adquisición de señales biomédicas," Tesis de pregrado, Bogotá, Pontificia Universidad Javeriana, 2017.

[12] "Desarrollo de herramientas de análisis de electrohisterográficas para el estudio de la respuesta mioeléctrica uterina a los fármacos de inducción del parto," Tesis de pregrado, Valencia, Universitat Politècnica de València, 2018.

[13] C. J. De Luca, Surface electromyography: Detection and recording, DelSys Incorporated, 2002.

[14] T. R. Cutmore y D. A. James, "Identifying and reducing noise in psychophysiological recordings," International journal of psychophysiology: official journal of the International Organization of Psychophysiology, vol. 32, n. ${ }^{\circ}$ 2, págs. 129-150, mayo de 1999.

[15] M. Hassan, J. Terrien, C. Muszynski, A. Alexandersson, C. Marque y B. Karlsson, "Better Pregnancy Monitoring Using Nonlinear Correlation," 
IEEE Transactions on Biomedical Engineering, vol. 60, n. o 4, págs. 1160-1166, abr. de 2012.

[16] Y. Ye Lin, J. Alberola Rubio, G. Prats Boluda, A. J. Perales Marin, D. Desantes y F. J. Garcia Casado, "Feasibility and Analysis of Bipolar Concentric Recording of Electrohysterogram with Flexible Active Electrode," Annals of Biomedical Engineering, vol. 43, n. ${ }^{\circ}$ 4, págs. 968-976, abr. de 2015.

[17] M. S. Jóhannsdóttir, The Effect of Different Electrode Design on the Electrohysterogram Signal," Tesis de pregrado, Reykjavik, Svansprent, 2015.

[18] P. Ren, S. Yao, J. Li, P. A. Valdés-Sosa y K. M. Kendrick, "Improved Prediction of Preterm Delivery Using Empirical Mode Decomposition Analysis of Uterine Electromyography Signals," PLoS ONE, vol. 10, n. ${ }^{\circ}$ 7, e0132116, jul. de 2015.

[19] H. De Lau, C. Rabotti, H. P. Oosterbaan, M. Mischi y G. S. Oei, "Study protocol: PoPE-Prediction of preterm delivery by electrohysterography," BMC Pregnancy Childbirth, vol. 14, n. ${ }^{\circ} 192,2014$.

[20] M. A. Chamorro Lucero, M. F. Acosta Romo y C. A. Quinayas Burgos, 'Análisis de señales electro-histerográficas (ehg) paradetectar cambios bioeléctricos en la actividad muscular uterina," de Perspectivas de Investigación e innovación interdisciplinar, Primera ed., L. E. Vera Hernández, Ed., San Juan de Pasto, Nariño: Editorial UNIMAR, 2018, pp. 409-422.

[21] R. Lopez y T. C. Davies, The effect of surface electromyography placement on muscle activation amplitudes and timing, Ottawa, Ontario, 2016.

[22] Globedia, "Monitorizacion fetal," 3 febrero 2016. [Online]. Available: http://co.globedia.com/monitorizacion-fetal. 